Erratum

\title{
GALNT6 Promotes Tumorigenicity and Metastasis of Breast Cancer Cell via $\beta$-catenin/MUCl-C Signaling Pathway: Erratum
}

\author{
Yingge Mao ${ }^{1,2,4 \#}$, Yuqi Zhang1,2\#, Sairong Fan ${ }^{1,2 \#, ~ L v a o ~ C h e n ~}{ }^{1,2}$, Lili Tang ${ }^{1,2}$, Xiaoming Chen ${ }^{1,2}$, Jianxin \\ $\mathrm{Lyu}^{2,3}$ \\ 1. Institute of Glycobiological Engineering, School of Laboratory Medicine and Life Sciences, Wenzhou Medical University, Wenzhou, Zhejiang, China \\ 2. Zhejiang Provincial Key Laboratory of Medical Genetics, Key Laboratory of Laboratory Medicine, Ministry of Education, China, School of Laboratory \\ Medicine and Life Sciences, Wenzhou Medical University, Wenzhou, Zhejiang, China \\ 3. Hangzhou Medical College, Hangzhou, Zhejiang, China \\ 4. Present address: The First Affiliated Hospital of Henan University \\ \#These authors contributed equally to this work.
}

$\triangle$ Corresponding author: Xiaoming Chen, Institute of Glycobiological Engineering/School of Laboratory Medicine and Life Sciences, Wenzhou Medical University, Wenzhou, Zhejiang, 325035, China. Tel: 086-577-86699651; Fax: 086-577-86689717; E-mail: xmc@wmu.edu.cn

(c) The author(s). This is an open access article distributed under the terms of the Creative Commons Attribution License (https://creativecommons.org/licenses/by/4.0/). See http://ivyspring.com/terms for full terms and conditions.

Published: 2021.11.11

Corrected article: Int J Biol Sci 2019; 15(1): 169-182. doi: 10.7150/ijbs.29048.

In our paper [1], the image of invaded MDA-MB-231 cells with empty vector transfection (shRNA-NC) in Figure 3C was mis-pasted. The image of invaded MDA-MB-231 cells (Mock) was accidentally used for "shRNA-NC" in Figure 3C. Neither the interpretation nor the conclusion of this work is affected by this error. Figure 3C should be corrected as follows.

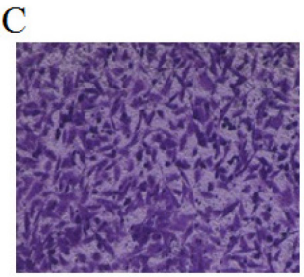

Mock

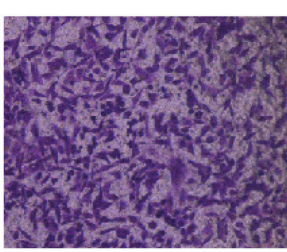

shRNA-NC

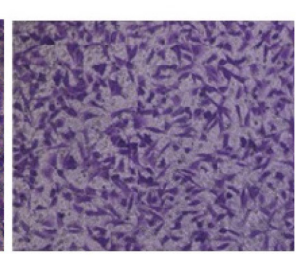

shRNA-T6

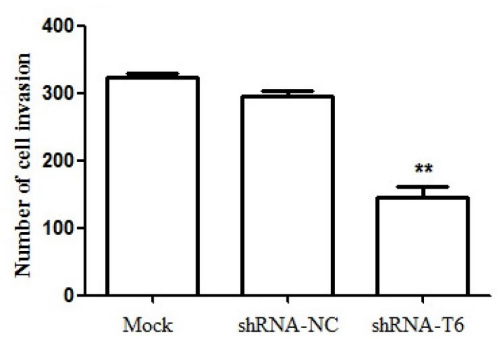

Figure 3. Down-regulation of GALNT6 inhibited the migration and invasion capacity of MDA-MB-231 cells. (A) Wound healing assay and quantification analysis showed that knockdown of GALNT6 significantly inhibited cell migration in MDA-MB-231 cells. (B) Transwell migration assay and quantification analysis showed that knockdown of GALNT6 significantly inhibited cell migration in MDA-MB-231 cells. (C) Matrigel invasion assay and quantification analysis showed that knockdown of GALNT6 greatly inhibited invasive abilities of MDA-MB-231 cells. (D-E) Nude mice $\left(n=5\right.$ per group) were injected with $1 \times 10^{7}$ MDA-MB-231 cells (Mock, shRNA-NC or shRNA-T6) via the venous plexus of the eye. After 4 weeks, the mice were sacrificed under anesthesia. (D) The lungs were subjected to Bouin's fixation and photographed. A representative of the experiments is shown. Visible lung metastases nudes were counted. (E) The sections of lungs were stained with H\&E. Data are expressed as means $\pm S E M$. $* P<0.05$ and $* * P<$ 0.01 .

\section{References}

[1] Mao Y, Zhang Y, Fan S, Chen L, Tang L, Chen X, et al. GALNT6 Promotes Tumorigenicity and Metastasis of Breast Cancer Cell via $\beta$-catenin/MUC1-C Signaling Pathway. Int J Biol Sci. 2019; 15(1):169-182. doi:10.7150/ijbs.29048. 